\title{
Comorbidities and Factors Determining Medical Expenses and Length of Stay for Admitted COVID-19 Patients in Korea
}

\author{
Su Yeon Jang ${ }^{1, *}$ \\ Jeong-Yeon Seon $\mathbb{1 D}^{1, *}$ \\ Seok-Jun Yoon ${ }^{2}$ \\ So-Youn Park ${ }^{3}$ \\ Seung Heon Lee $\mathbb{D}^{4, *}$ \\ In-Hwan $\mathrm{Oh}^{1}{ }^{1} *$
}

'Department of Preventive Medicine, School of Medicine, Kyung Hee University, Seoul, South Korea; ${ }^{2}$ Department of Preventive Medicine, Korea University College of Medicine, Seoul, South Korea; ${ }^{3}$ Department of Medical Education and Humanities, School of Medicine, Kyung Hee University, Seoul, South Korea; ${ }^{4}$ Division of Pulmonary, Sleep, and Critical Care Medicine, Department of Internal Medicine, Korea University Ansan Hospital, Ansan, South Korea

*These authors contributed equally to this work
Correspondence: In-Hwan Oh Department of Preventive Medicine, School of Medicine, Kyung Hee University, 26 Kyungheedae-ro, Dongdaemun-gu, Seoul, 02447, South Korea

Email parenchyme@gmail.com

Seung Heon Lee

Division of Pulmonary, Sleep, and Critical Care Medicine, Department of Internal Medicine, Korea University Ansan Hospital, 123 Jeokgeum-ro, Danwon-gu, Ansan, Gyeonggi-do, 15355, South Korea Email lee-sh@korea.ac.kr
Purpose: No previous investigations of coronavirus disease 2019 (COVID-19) have estimated medical expenses, length of stay, or factors influencing them using administrative datasets. This study aims to fill this research gap for the Republic of Korea, which has over 10,000 confirmed COVID-19 cases.

Patients and Methods: Using the nationwide health insurance claims data of 7590 confirmed COVID-19 patients, we estimated average medical expenses and inpatient days per patient, and performed multivariate negative binomial, and gamma regressions to determine influencing factors for higher outcomes.

Results: According to the results, COVID-19 patients with history of ICU admission, chest CT imaging, lopinavir/ritonavir and hydroxychloroquine use stayed longer in the hospital and spent more on medical expenses, and anti-hypertensive drugs were insignificantly associated with the outcomes. Female patients stayed longer in the hospital in the over 65 age group but spent less in medical expenses that the 20-39 group. In the 40-69 age group, patients with health insurance stayed longer in the hospital and spent more on medical expenses than those aged over 65 years. Comorbidities did not affect outcomes in most age groups.

Conclusion: In summary, contrary to popular beliefs, medical expenses and length of hospitalization were mostly influenced by age, and not by comorbidities, anti-viral, or antihypertensive drugs. Thus, responses should focus on infection prevention and control rather than clinical countermeasures.

Keywords: medical costs, length of stay in hospital, comorbidities, COVID-19, Republic of Korea

\section{Introduction}

In the Republic of Korea (also known as South Korea; henceforth, Korea), since the first confirmed case of coronavirus 2019 (COVID-19) was reported on January 20, $2020,{ }^{1}$ there have been a total of 22,285 confirmed cases as of September 14, 2020. ${ }^{2}$ Among the infected patients, nearly $40 \%$ were confirmed in the first two months of the pandemic, with 8652 (38.8\%) individuals confirmed of the disease as of March 20, 2020. ${ }^{3}$ According to the previous findings, patients from the early phase of the pandemic had higher case fatality rates if they were above 70 years of age and male compared to younger individuals and female patients; the highest proportion of confirmed cases were female (61.5\%) and those in their $20 \mathrm{~s}(27.3 \%)$; in deceased cases, males $(54.3 \%)$ were higher in proportion than females and the 
fatality was highest in the age group of 80 years or above $(35.1 \%)$, indicating high fatality among the elderly. ${ }^{3-5}$

Korea is one of the world's fastest ageing societies. Among a total of 52 million people within its territory, over $24 \%$ are aged 60 or above ( $45 \%$ men; $55 \%$ women), which is expected to increase to over $40 \%$ of the total population by $2047 .{ }^{6,7}$ Older adults are especially more prone to some of the chronic conditions that are common comorbidities of COVID-19, such as cardiovascular risk factors and the chronic obstructive pulmonary disease (COPD). ${ }^{8,9}$ In particular, the prevalence of cardiovascular conditions in the Korean female population shows a dramatic increase, from under $10 \%$ for women in their 30 s to over $50 \%$ in their $70 \mathrm{~s}$; a similar trend is observed regarding the prevalence of COPD in the male population. ${ }^{8,9}$ Considering the rapid ageing trend of the Korean society and the high prevalence of predisposing risk factors in the elderly population, in addition to the nonnegligible mortality and fatality due to COVID-19, the burden posed by the novel infection cannot be overlooked.

Although there have been some clinical studies of COVID-19 in Korea, little research has estimated the risk factors for severe clinical course. The scope of clinical manifestations and the spectrum of COVID-19 has a wide range, from asymptomatic infections to severe states with respiratory failure demanding intensive critical care with mechanical ventilation, renal replacement therapy, and extracorporeal membrane oxygenation; rapid clinical deterioration could be found unexpectedly in admitted patients within days. ${ }^{10}$ Previous studies in China and the United States (US) have reported that treatment outcomes are associated with older age and comorbid conditions such as hypertension, diabetes, coronary heart disease, and chronic obstructive pulmonary disease (COPD). ${ }^{11-16}$

Moreover, severe illness influences not only direct clinical outcomes but also higher medical expenses and longer duration of hospitalization of patients. For instance, direct medical costs for hospitalized patients with influenza-related diseases in China have been reported to be higher among patients with underlying medical conditions or those in severer states than among those without a preceding medical history or having a mild state of the disease. $^{17,18}$ In addition, in the case of severe acute respiratory syndrome (SARS), patients who were older had a longer hospital stay while the hospitalization period in deceased cases was shorter among older than younger people. $^{19}$ Nonetheless, only a limited number of studies have investigated the direct medical costs and the length of stay in hospital among COVID-19 patients.

In Korea, all residents benefit from the universal healthcare system through the National Health Insurance (NHI) and the Medical Aid Program, in which claimed fees are reimbursed to providers after review by the Health Insurance Review and Assessment Service (HIRA). ${ }^{20}$ Thus, data on medical resource utilization of the entire Korean population are produced and collected through benefit claims and reimbursement procedures, which enables the investigation of the health status of the whole population. The South Korean government has shared the world's first de-identified COVID-19 nationwide insurance claims data provided by HIRA through the COVID-19 International Research project. ${ }^{21}$ In particular, the coverage of COVID-19 is entirely covered by the national health insurance program due to the application of exempted insurance fees in response to the pandemic crisis. Thus, data from the COVID-19 International Research project include information on screening tests, diagnosis, treatments, and outcomes of the disease in all residents within Korea.

Therefore, this study aims to estimate direct medical expenses and the length of hospitalization due to COVID19 , as well as to determine their influencing factors. In this study, we utilize the health insurance fee claims data from the COVID-19 International Research project of Korea to examine the detailed costs and the length of stay in the early phase (ie, the first 12 weeks) of the pandemic; in particular, we investigate the details of demographic and clinical characteristics, including comorbid conditions, ICU admissions, and such treatments as the use of medication or mechanical ventilators. Thereafter, we analyze the risk factors of having higher medical expenditures or longer inpatient stays by each of the demographic and clinical features of Korean patients with COVID-19.

\section{Patients and Methods Study Design and Participants}

This study used insurance benefits claims data provided by HIRA through the COVID-19 International Research project of the Korean government. For the COVID-19 International Research project, HIRA provides the insurance benefit claims data relevant to COVID-19 and the history of medical service use for the past three years (January 2017 to February 2020) for all COVID-19 patients of South Korea as of May 15, 2020. ${ }^{21}$ The 
Health Insurance Review \& Assessment Service identified 7590 confirmed patients by connecting the claims with the list of confirmed COVID-19 patients from the Korea Disease Control and Prevention Agency (KCDA). This information was also provided in the COVID-19 patient data set. The 7590 confirmed COVID-19 patients were defined as targets for analysis.

\section{Data Collection}

This study collected the following insurance claims data: demographic information (sex, age, healthcare coverage, and provider region), comorbidities (cancer, COPD, ischemic heart disease, hypertension, and diabetes), use of renin-angiotensin-aldosterone system (RAAS) inhibitor anti-hypertensive drugs (angiotensin-converting-enzyme inhibitor [ACEI] and angiotensin II receptor blocker [ARB]; detailed descriptions with the anatomical therapeutic chemical classification codes in Supplementary Table 1), treatments or healthcare resource utilization (ICU admission, mechanical ventilation, chest computed tomography [CT], and use of the medications interferon, lopinavir/ritonavir [typically Kaletra], and hydroxychloroquine), direct medical expenses (testing, treatment, and pharmaceutical costs occurred in patients with the major diagnosis of COVID-19), and the length of stay in hospital (days spent in hospital). We considered healthcare coverage (health insurance and medical aid beneficiaries) as one of the demographic variables to assess patients' financial status, and the utilized provider's region (Daegu, Gyeongsangbuk-do [Gyeongbuk], and others) to compare the origins of community-acquired infections (ie, Daegu and Gyeongbuk) among regions. Comorbidities were defined as conditions from 1 year before the diagnosis of COVID-19. In addition, to identify the risk of RAAS inhibitors in COVID-19 patients, prescription of ACEI and ARB within 6 weeks of the COVID-19 test was defined as the use of such drugs and investigated.

\section{Statistical Analysis}

We calculated descriptive statistics about length of stay and medical expenses by demographical characteristics of COVID-19 patients. This study considered sex, age, provider region, comorbid conditions, and treatments in hospital for patients with COVID-19 as demographical characteristics. Then, we confirmed the differences in costs and duration according to demographical characteristics by using univariate generalized linear regression analysis. To compare differences in length of stay, univariate negative binomial regression analysis was used. Gamma regression analysis was performed to compare medical expenses. Multivariate generalized linear regressions were performed to analyze the influencing factors regarding medical expenses and the length of stay in hospital for all patients aggregated and by each age group. Variables included in the regression models were demographics, comorbidities, anti-hypertensive drug use, healthcare resource utilization, and treatments that included the use of medications. Negative binomial regressions were performed to analyze the length of hospital stay, and gamma regressions were performed for medical expenses.

In this study, we chose to present medical expenses based on United States dollars (USD) rather than Korean won (KRW). We used the basic exchange rate for KRW per USD on May 15, 2020, which was USD $1=$ KRW $1227.90 .^{22}$ All statistical analyses were performed with SAS 9.4 and the significance of all statistical tests was determined using the 0.05 level.

\section{Ethics Statement}

This study was approved by Kyung Hee University's Institutional Review Board (IRB No. KHSIRB-20-164). Informed consent was not required owing to the use of deidentified data provided by HIRA.

\section{Results}

\section{Demographic and Clinical Characteristics of COVID-19 Patients}

This study included 7590 patients confirmed with COVID19 in Korea. Table 1 presents the differences by the length of stay and medical expenses by the demographic and clinical characteristics of COVID-19 patients. Differences by the mean length of stay by sex is not significantly confirmed ( $p$-value $=0.222)$, but males $(4633 \pm 5837$ USD) show a higher mean of medical expenses than females (4067 \pm 5335 USD). Means of length of stay and medical expenses increase with advancing age ( $p$-value $<0.001$ ). Association between healthcare coverage and length of stay and medical expenses is not statistically significant. Length of stay of Daegu (23.0 16.4 days) is the longest, but the highest medical expense is observed in others (4497 \pm 5506 USD).

The greater number of comorbidities also correlates with longer durations and higher costs $(p$-value $<0.001)$. 
Table I Length of Stay and Medical Expenses by Characteristics of COVID-I 9 Patients

\begin{tabular}{|c|c|c|c|c|c|}
\hline & \multicolumn{5}{|c|}{ COVID-I9 Patients $(n=7590)$} \\
\hline & \multirow[t]{2}{*}{$\mathbf{n}$} & \multicolumn{2}{|c|}{ Length of Stay (Days) } & \multicolumn{2}{|c|}{ Medical Expenses (USD) } \\
\hline & & Mean \pm SD & $p$-value ${ }^{a}$ & Mean士SD & $p$-value \\
\hline \multicolumn{6}{|l|}{ Sex } \\
\hline Male & 3095 & $21.6 \pm 14.1$ & 0.222 & $4633 \pm 5837$ & $<0.001$ \\
\hline Female & 4495 & $22.0 \pm 14.7$ & & $4067 \pm 5335$ & \\
\hline \multicolumn{6}{|l|}{ Age (years) } \\
\hline $0-19$ & 431 & $19.1 \pm 10.6$ & $<0.001$ & $2927 \pm 3101$ & $<0.001$ \\
\hline $20-39$ & 2629 & $19.1 \pm 11.2$ & & $3205 \pm 3315$ & \\
\hline $40-64$ & 3165 & $22.4 \pm 14.9$ & & $4432 \pm 5471$ & \\
\hline $65+$ & 1365 & $26.9 \pm 17.9$ & & $6523 \pm 8365$ & \\
\hline \multicolumn{6}{|l|}{ Healthcare coverage } \\
\hline Medical aid & 629 & $21.6 \pm 17.0$ & 0.673 & $4035 \pm 5433$ & 0.129 \\
\hline Health insurance & 6961 & $21.9 \pm 14.2$ & & $4322 \pm 5562$ & \\
\hline \multicolumn{6}{|l|}{ Provider region } \\
\hline Daegu & 2696 & $23.0 \pm 16.4$ & $<0.001$ & $4184 \pm 5527$ & 0.006 \\
\hline Gyeongsangbok-do & $142 \mid$ & $21.8 \pm 13.0$ & & $4026 \pm 5696$ & \\
\hline Others & 3473 & $21.0 \pm 13.3$ & & $4497 \pm 5506$ & \\
\hline \multicolumn{6}{|c|}{ Number of comorbidities ${ }^{c}$} \\
\hline 0 & 5427 & $20.7 \pm 13.4$ & $<0.001$ & $378 I \pm 4595$ & $<0.001$ \\
\hline 1 & 1694 & $24.1 \pm 15.8$ & & $5429 \pm 7358$ & \\
\hline 2 & 403 & $26.8 \pm 17.8$ & & $6196 \pm 7039$ & \\
\hline $3+$ & 66 & $26.2 \pm 17.9$ & & $6190 \pm 6195$ & \\
\hline \multicolumn{6}{|l|}{ Cancer } \\
\hline No & 7252 & $21.7 \pm 14.3$ & $<0.001$ & $4223 \pm 5531$ & $<0.001$ \\
\hline Yes & 338 & $26.0 \pm 16.1$ & & $5902 \pm 5757$ & \\
\hline \multicolumn{6}{|l|}{ COPD } \\
\hline No & 7031 & $21.8 \pm 14.4$ & $<0.001$ & $4273 \pm 5577$ & 0.116 \\
\hline Yes & 559 & $23.0 \pm 14.3$ & & $4607 \pm 5215$ & \\
\hline \multicolumn{6}{|c|}{ Ischemic heart disease } \\
\hline No & 7352 & $21.7 \pm 14.3$ & $<0.001$ & $4237 \pm 5494$ & $<0.001$ \\
\hline Yes & 238 & $26.3 \pm 18.5$ & & $6177 \pm 6855$ & \\
\hline \multicolumn{6}{|l|}{ Hypertension } \\
\hline No & 6627 & $21.4 \pm 14.0$ & $<0.001$ & $404 I \pm 4867$ & $<0.001$ \\
\hline Yes & 963 & $25.2 \pm 16.9$ & & $6068 \pm 874 \mid$ & \\
\hline \multicolumn{6}{|l|}{ Diabetes } \\
\hline No & 6986 & $21.5 \pm \mid 4.1$ & $<0.001$ & $4162 \pm 5415$ & $<0.001$ \\
\hline Yes & 604 & $25.8 \pm 17.6$ & & $5867 \pm 6755$ & \\
\hline \multicolumn{6}{|l|}{ ICU admission } \\
\hline No & 6744 & $20.9 \pm 13.7$ & $<0.001$ & $3335 \pm 3134$ & $<0.001$ \\
\hline Yes & 846 & $29.4 \pm 17.8$ & & ||$, 974 \pm||, 49 \mid$ & \\
\hline \multicolumn{6}{|l|}{ Chest CT } \\
\hline No & 4914 & $19.3 \pm 13.4$ & $<0.001$ & $3065 \pm 4082$ & $<0.001$ \\
\hline Yes & 2676 & $26.6 \pm|5|$. & & $6562 \pm 6994$ & \\
\hline
\end{tabular}

(Continued) 
Table I (Continued).

\begin{tabular}{|c|c|c|c|c|c|}
\hline & \multicolumn{5}{|c|}{ COVID-19 Patients $(n=7590)$} \\
\hline & \multirow[t]{2}{*}{$\mathbf{n}$} & \multicolumn{2}{|c|}{ Length of Stay (Days) } & \multicolumn{2}{|c|}{ Medical Expenses (USD) } \\
\hline & & Mean $\pm S D$ & $p$-value ${ }^{a}$ & Mean $\pm S D$ & $p$-value ${ }^{b}$ \\
\hline \multicolumn{6}{|c|}{ Mechanical ventilation } \\
\hline No & 7469 & $21.7 \pm 14.3$ & $<0.001$ & $3953 \pm 3942$ & $<0.001$ \\
\hline Yes & 121 & $29.2 \pm 19.9$ & & $25,555 \pm 22,783$ & \\
\hline \multicolumn{6}{|c|}{ ACEI use } \\
\hline No & 7563 & $21.9 \pm 14.4$ & 0.470 & $4298 \pm 5557$ & 0.961 \\
\hline Yes & 27 & $24.0 \pm 17.7$ & & $4255 \pm 3851$ & \\
\hline \multicolumn{6}{|c|}{ ARB use } \\
\hline No & 6843 & $21.4 \pm 13.9$ & $<0.001$ & $4064 \pm 4941$ & $<0.001$ \\
\hline Yes & 747 & $26.6 \pm 18.0$ & & $6442 \pm 9193$ & \\
\hline \multicolumn{6}{|c|}{ Lopinavir/ritonavir use } \\
\hline No & 4927 & $18.6 \pm 12.7$ & $<0.001$ & $2856 \pm 3267$ & $<0.001$ \\
\hline Yes & 2663 & $27.9 \pm 15.5$ & & $6966 \pm 7559$ & \\
\hline \multicolumn{6}{|c|}{ HCQ use } \\
\hline No & 7527 & $21.8 \pm 14.4$ & $<0.001$ & $3410 \pm 4206$ & $<0.001$ \\
\hline Yes & 63 & $25.0 \pm 17.7$ & & $6603 \pm 7587$ & \\
\hline \multicolumn{6}{|c|}{ Interferon use } \\
\hline No & 7527 & $21.8 \pm 14.4$ & $<0.001$ & $4249 \pm 5479$ & $<0.001$ \\
\hline Yes & 63 & $25.0 \pm 17.7$ & & $10,108 \pm 9686$ & \\
\hline
\end{tabular}

Notes: ${ }^{\mathrm{a}} \mathrm{p}$-value for univariate negative binomial regression analysis. ${ }^{\mathrm{b}} \mathrm{p}$-value for univariate gamma regression analysis. ${ }^{\mathrm{c}}$ Cancer, chronic obstructive pulmonary disease, ischemic heart disease, hypertension, diabetes.

Abbreviations: SD, standard deviation; COPD, chronic obstructive pulmonary disease; ICU, intensive care unit; CT, computed tomography; ACEl, angiotensin-convertingenzyme inhibitor; ARB, angiotensin II receptor blocker; HCQ, hydroxychloroquine.

COVID-19 patients with cancer (26.0 16.1 days), COPD (23.0 \pm 14.3 days), ischemic heart disease ( $26.3 \pm 18.5$ days), hypertension (25.2 \pm 16.9 days), and diabetes $(25.8 \pm 17.6$ days) present longer length of stay than those without these diseases. COVID-19 patients with cancer (5902 \pm 5757 USD), ischemic heart disease (6177 \pm 6855 USD), hypertension (6068 \pm 8741 USD), diabetes (5867 \pm 6755 USD) show higher medical expenses, but the difference in COPD is not confirmed.

COVID-19 patients who used ICU (29.4 \pm 17.8 days, $11,974 \pm 11,491$ USD), chest CT imaging (26.6 \pm 15.1 days, $6562 \pm 6994$ USD), mechanical ventilators $(29.2 \pm 19.9$ days, 25,555 $\pm 22,783$ USD) stayed longer in the hospital, and incurred higher medical expenses. Means of length of stay (24.0 \pm 17.7 days) and medical expenses $(4255 \pm 3851$ USD) of COVID-19 patients who used ACEI are not significantly different from those who did not use the

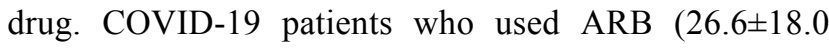
days, $6442 \pm 9193$ USD), lopinavir/ritonavir $(27.9 \pm 15.5$ days, $6966 \pm 7559$ USD), HCQ (25.0 \pm 17.7 days, 6603 \pm 7587 USD), and interferon (25.0 \pm 17.7 days, 10,108 \pm 9686 USD) stayed longer in the hospital, and incurred higher medical expenses than those who did not, and we could confirm statistical differences ( $p$-value $<0.001)$.

\section{Influencing Factors of Medical Expenses and Length of Stay in Hospital Due to COVID-19}

In Table 1, we found that age, provider region, number of comorbidities, comorbidities (cancer, COPD, ischemic heart disease, hypertension, diabetes), medical equipment use (ICU, chest CT, mechanical equipment), and drug use (ARB, lopinavir/ritonavir, HCQ, interferon) affected length of stay. Sex, age, provider region, number of comorbidities, comorbidities (cancer, ischemic heart disease, hypertension, diabetes), medical equipment use (ICU, chest CT, mechanical equipment), and drug use (ARB, lopinavir/ritonavir, HCQ, interferon) had an association with medical expenses in COVID-19 patients. 
Table 2 Results of Generalized Linear Regression Analyses for Length of Stay and Medical Expenses of COVID-19 Patients

\begin{tabular}{|c|c|c|c|c|c|c|}
\hline & \multicolumn{6}{|c|}{ COVID-19 Patients ( $n=7590)$} \\
\hline & \multicolumn{3}{|c|}{ Length of Stay (Days) } & \multicolumn{3}{|c|}{ Medical Expenses (USD) } \\
\hline & Estimates & SE & $p$-value & Estimates & SE & $p$-value \\
\hline \multicolumn{7}{|c|}{ Sex $($ reference $=$ male $)$} \\
\hline Female & 0.03 & 0.01 & 0.045 & -0.02 & 0.02 & 0.282 \\
\hline \multicolumn{7}{|c|}{ Age (reference $=0-19$ years) } \\
\hline $20-39$ years & -0.08 & 0.03 & 0.012 & -0.11 & 0.05 & 0.022 \\
\hline $40-64$ years & -0.06 & 0.03 & 0.088 & -0.07 & 0.05 & 0.156 \\
\hline $65+$ years & -0.03 & 0.04 & 0.455 & -0.07 & 0.05 & 0.186 \\
\hline \multicolumn{7}{|c|}{ Healthcare coverage (reference $=$ medical aid) } \\
\hline Health insurance & 0.07 & 0.03 & 0.009 & 0.16 & 0.04 & $<0.001$ \\
\hline \multicolumn{7}{|c|}{ Provider region (reference $=$ others) } \\
\hline Daegu & -0.01 & 0.02 & 0.448 & -0.12 & 0.02 & $<0.001$ \\
\hline Gyeongsangbok-do & 0.03 & 0.02 & 0.100 & 0.05 & 0.03 & 0.101 \\
\hline \multicolumn{7}{|c|}{ Cancer $($ reference $=\mathbf{n o})$} \\
\hline Yes & 0.05 & 0.03 & 0.185 & 0.07 & 0.05 & 0.163 \\
\hline \multicolumn{7}{|c|}{ COPD (reference $=$ no) } \\
\hline Yes & 0.00 & 0.03 & 0.935 & 0.06 & 0.04 & 0.130 \\
\hline \multicolumn{7}{|c|}{ Ischemic heart disease (reference $=$ no) } \\
\hline Yes & 0.02 & 0.04 & 0.680 & -0.01 & 0.06 & 0.859 \\
\hline \multicolumn{7}{|c|}{ Hypertension (reference $=$ no) } \\
\hline Yes & -0.01 & 0.03 & 0.812 & 0.04 & 0.04 & 0.342 \\
\hline \multicolumn{7}{|c|}{ Diabetes (reference $=$ no) } \\
\hline Yes & -0.01 & 0.03 & 0.817 & 0.00 & 0.04 & 0.913 \\
\hline \multicolumn{7}{|c|}{ ICU admission (reference $=$ no) } \\
\hline Yes & 0.22 & 0.02 & $<0.001$ & 0.92 & 0.04 & $<0.001$ \\
\hline \multicolumn{7}{|c|}{ Chest CT (reference $=$ no) } \\
\hline Yes & 0.19 & 0.02 & $<0.001$ & 0.52 & 0.02 & $<0.001$ \\
\hline \multicolumn{7}{|c|}{ ACEI use (reference $=$ no) } \\
\hline Yes & -0.02 & 0.12 & 0.853 & -0.23 & 0.18 & 0.196 \\
\hline \multicolumn{7}{|c|}{ ARB use (reference $=$ no) } \\
\hline Yes & 0.05 & 0.03 & 0.065 & -0.02 & 0.04 & 0.681 \\
\hline \multicolumn{7}{|c|}{ Lopinavir/ritonavir use $($ reference $=$ no) } \\
\hline Yes & 0.28 & 0.02 & $<0.001$ & 0.60 & 0.02 & $<0.001$ \\
\hline \multicolumn{7}{|c|}{ HCQ use (reference $=$ no) } \\
\hline Yes & 0.25 & 0.02 & $<0.001$ & 0.35 & 0.03 & $<0.001$ \\
\hline
\end{tabular}

Notes: a Multivariate negative binomial regression model including mechanical ventilator application and interferon use. ${ }^{b}$ Multivariate gamma regression model including mechanical ventilator application and interferon use.

Abbreviations: SE, standard error; COPD, chronic obstructive pulmonary disease; ICU, intensive care unit; $\mathrm{CT}$, computed tomography; ACEI, angiotensin-convertingenzyme inhibitor; ARB, angiotensin II receptor blocker; HCQ, hydroxychloroquine.

Table 2 shows the adjusted results from negative binomial and gamma regression models analyzing the influencing factors of length of hospital stay and medical expenses.
In the negative binomial regression model, sex, age, healthcare coverage, ICU admission, chest CT, lopinavir/ ritonavir use, and HCQ use shows an association with 
duration in hospital. Female patients with COVID-19 stayed 3\% longer in the hospital than males. In the 20-39 age group, length of stay had significant difference ( $p$-value $=0.012)$, and they stayed $8 \%$ less than the $0-19$ group. Regarding duration in hospital to treat COVID-19, patients with medical aid stayed $7 \%$ longer than those with health insurance. COVID-19 patients who were admitted to ICU stayed $22 \%$ longer in hospital than those who were not admitted, and those who underwent a chest CT stayed $19 \%$ longer. Patients who used lopinavir/ritonavir and HCQ were admitted to the hospital longer than those who did not use these drugs.

In the gamma regression model, medical expenses, age, healthcare coverage, provider region, ICU admission, chest CT, lopinavir/ritonavir use, and HCQ use present an association with costs to treat COVID-19. In the 20-39 age group, medical expenses had significant difference $(p$-value $=0.022)$, $11 \%$ lower than the $0-19$ group. Medical aid patients spend $16 \%$ higher on medical expenses than those with health insurance, and Daegu patients spend $12 \%$ less on the costs to treat COVID-19. Medical equipment usage results in higher medical expenditure, and COVID-19 patients who were admitted to ICU paid $92 \%$ more than those who were not. Those who underwent chest CT paid 52\% more than those who did not. Medical expenses for patients who used lopinavir/ritonavir and HCQ were $60 \%$ and 35\% higher, respectively, than those who did not use these drugs. Results from regression analyses about length of stay and medical expenses considered the number of comorbidities instead of each comorbidity separately, and presented overall similar trends (see Supplementary Table 2).

Table 3 shows the results of age-stratified negative binomial regression analyses about COVID-19 treatment duration. In the 20-39 age group, healthcare utilization from providers located in Gyeongbuk province, ICU admission, chest CT imaging, lopinavir/ritonavir use, and HCQ use were significantly associated with longer length of stay in hospital. In the 40-64 age group, health insurance status, healthcare utilization from providers located in Daegu, ICU admission, chest CT imaging, lopinavir/ritonavir use, and HCQ use was significantly associated with the duration of COVID-19 treatment in hospital. In the over 65 age group, female, ICU admission, chest CT imaging, lopinavir/ritonavir use, and HCQ use resulted in longer hospital stay. Results from the age-stratified negative binomial regression analyses about length of stay considered the number of comorbidities instead of each comorbidity separately, and presented overall similar trends (see Supplementary Table 3).
Table 4 shows the results of age-stratified negative binomial regression analyses regarding costs to treat COVID-19. In the 20-39 age group, male, healthcare utilization from providers located in Gyeongbuk province, ICU admission, chest CT imaging, lopinavir/ritonavir use, and HCQ use were significantly associated with higher medical expenses. In the 40-64 age group, health insurance status, COPD, ICU admission, chest CT imaging, lopinavir/ritonavir use, and HCQ use resulted in significantly higher costs to treat COVID-19. Patients who used healthcare from providers located in Daegu province spent on $12 \%$ less on medical expenses than others. In the over 65 age group, health insurance patients, ICU admission, chest CT imaging, lopinavir/ritonavir use, and HCQ use resulted in higher medical expenses. Patients who used healthcare from providers located in Daegu province spent $35 \%$ lower on medical expenses than others. Results from the age-stratified gamma regression analyses about medical expenditures considered number of comorbidities instead of each comorbidity separately and presented overall similar trends (see Supplementary Table 4 and supplementary table 5).

\section{Discussion}

This study estimated the cost of direct medical expenses and the length of stay in hospital among COVID-19 patients in Korea, using nationwide health insurance benefit claims data. According to our results, the average cost of medical expenses was USD 1193.7 and the average length of hospital stay was 5.5 days per patient. When observed by sex and age group, the average medical expenses for both male and female patients were higher for older ages. The average length of stay in hospital also tended to be higher for older ages, except for the group aged 65 years or above, which had a shorter hospital stay than the group aged 40-64 years. Our results show a higher burden of COVID-19 among older patients through increased medical expenses and length of hospitalization in accordance with previous reports on inhospital deaths and ICU admissions of the elderly. ${ }^{12,15}$ An exception is shorter inpatient stays for those aged 65 years or above, possibly because there are fewer survivors in this group. ${ }^{15,16}$ Especially in cases where medical demand surges significantly and overwhelms health infrastructure, chances are high that older patients fall victim to the lack of capacity to provide adequate care for every patient. 
Table 3 Results of Age-Stratified Negative Binomial Regression Analyses for Length of Stay of COVID-19 Patients

\begin{tabular}{|c|c|c|c|c|c|c|c|c|c|}
\hline & \multicolumn{9}{|c|}{ Negative Binomial Regression Model for Length of Stay ${ }^{\text {a }}$} \\
\hline & \multicolumn{3}{|c|}{ 20-39 Years $(n=2629)$} & \multicolumn{3}{|c|}{$40-64$ Years $(n=3165)$} & \multicolumn{3}{|c|}{$\geq 65$ Years $(n=1365)$} \\
\hline & Estimates & SE & $p$-value & Estimates & SE & $p$-value & Estimates & SE & $p$-value \\
\hline Sex $($ reference $=$ male $)$ & & & & & & & & & \\
\hline Female & 0.00 & 0.02 & 0.943 & 0.04 & 0.02 & 0.085 & 0.11 & 0.04 & 0.003 \\
\hline $\begin{array}{l}\text { Healthcare coverage (reference = } \\
\text { medical aid) }\end{array}$ & & & & & & & & & \\
\hline Health insurance & -0.04 & 0.07 & 0.536 & 0.10 & 0.04 & 0.005 & 0.08 & 0.06 & 0.146 \\
\hline Provider region (reference $=$ others) & & & & & & & & & \\
\hline Daegu & -0.08 & 0.03 & 0.003 & 0.02 & 0.03 & 0.343 & 0.00 & 0.05 & 0.996 \\
\hline Gyeongsangbok-do & 0.07 & 0.03 & 0.023 & -0.03 & 0.03 & 0.318 & 0.05 & 0.06 & 0.413 \\
\hline Cancer $($ reference $=$ no $)$ & & & & & & & & & \\
\hline Yes & 0.05 & 0.12 & 0.659 & 0.01 & 0.05 & 0.863 & 0.11 & 0.06 & 0.077 \\
\hline COPD $($ reference $=$ no $)$ & & & & & & & & & \\
\hline Yes & 0.00 & 0.05 & 0.982 & 0.01 & 0.04 & 0.741 & -0.04 & 0.06 & 0.462 \\
\hline Ischemic heart disease (reference $=$ no) & & & & & & & & & \\
\hline Yes & 0.15 & 0.19 & 0.423 & 0.05 & 0.07 & 0.437 & -0.02 & 0.06 & 0.789 \\
\hline Hypertension $($ reference $=$ no) & & & & & & & & & \\
\hline Yes & -0.01 & 0.14 & 0.920 & 0.01 & 0.04 & 0.848 & -0.04 & 0.04 & 0.331 \\
\hline Diabetes (reference $=$ no) & & & & & & & & & \\
\hline Yes & -0.14 & 0.14 & 0.314 & 0.06 & 0.04 & 0.114 & -0.08 & 0.05 & 0.089 \\
\hline ICU admission (reference $=$ no) & & & & & & & & & \\
\hline Yes & 0.16 & 0.04 & $<0.001$ & 0.22 & 0.04 & $<0.001$ & 0.29 & 0.05 & $<0.001$ \\
\hline Chest CT (reference $=$ no) & & & & & & & & & \\
\hline Yes & 0.23 & 0.03 & $<0.001$ & 0.17 & 0.02 & $<0.001$ & 0.12 & 0.04 & 0.003 \\
\hline ACEI use (reference $=$ no) & & & & & & & & & \\
\hline Yes & - & - & - & -0.32 & 0.23 & 0.163 & 0.08 & 0.16 & 0.611 \\
\hline ARB use (reference $=$ no) & & & & & & & & & \\
\hline Yes & 0.11 & 0.18 & 0.518 & 0.05 & 0.04 & 0.217 & 0.06 & 0.05 & 0.212 \\
\hline Lopinavir/ritonavir use (reference $=$ no) & & & & & & & & & \\
\hline Yes & 0.26 & 0.03 & $<0.001$ & 0.33 & 0.02 & $<0.001$ & 0.23 & 0.04 & $<0.001$ \\
\hline HCQ use (reference $=$ no) & & & & & & & & & \\
\hline Yes & 0.19 & 0.03 & $<0.001$ & 0.25 & 0.03 & $<0.001$ & 0.30 & 0.04 & $<0.001$ \\
\hline
\end{tabular}

Note: aMultivariate negative binomial regression model including mechanical ventilator application and interferon use.

Abbreviations: SE, standard error; COPD, chronic obstructive pulmonary disease; ICU, intensive care unit; CT, computed tomography; ACEI, angiotensin-convertingenzyme inhibitor; ARB, angiotensin II receptor blocker; HCQ, hydroxychloroquine.

Average cost of direct medical expenses among COVID-19 patients in this study (USD 1193.7) was estimated to be lower than that of pneumonia treatment (USD 1782) and Middle East respiratory syndrome (MERS) (male patients, USD 2827; female patients, USD 2326) ${ }^{23,24}$ Meanwhile, the average cost of direct medical expenses among COVID-19 patients in this study was estimated to be higher than that due to respiratory diseases such as COPD (USD 1000) and asthma (USD 227) in Korea. ${ }^{25,26}$ Furthermore, according to our study results, the average length of stay in hospital was 5.5 days for COVID-19 patients, which is shorter than that of pneumonia (9.0 days) or MERS patients (male patients, 10 days; female patients, 9 days) but longer than that of COPD (1.8 
Table 4 Results of Age-Stratified Gamma Regression Analyses for Medical Expenses of COVID-19 Patients

\begin{tabular}{|c|c|c|c|c|c|c|c|c|c|}
\hline & \multicolumn{9}{|c|}{ Gamma Regression Model for Medical Expenses ${ }^{\text {a }}$} \\
\hline & \multicolumn{3}{|c|}{ 20-39 Years $(n=2629)$} & \multicolumn{3}{|c|}{$40-64$ Years $(n=3165)$} & \multicolumn{3}{|c|}{$\geq 65$ Years $(n=1365)$} \\
\hline & Estimates & SE & p-value & Estimates & SE & p-value & Estimates & SE & p-value \\
\hline Sex $($ reference $=$ male $)$ & & & & & & & & & \\
\hline Female & -0.08 & 0.04 & 0.025 & 0.01 & 0.03 & 0.701 & 0.02 & 0.04 & 0.618 \\
\hline $\begin{array}{l}\text { Healthcare coverage (reference = } \\
\text { medical aid) }\end{array}$ & & & & & & & & & \\
\hline Health insurance & 0.10 & 0.11 & 0.400 & 0.16 & 0.05 & 0.002 & 0.13 & 0.06 & 0.043 \\
\hline Provider region (reference $=$ others) & & & & & & & & & \\
\hline Daegu & 0.00 & 0.04 & 0.954 & -0.12 & 0.04 & 0.001 & -0.35 & 0.06 & $<0.001$ \\
\hline Gyeongsangbok-do & 0.10 & 0.05 & 0.043 & -0.01 & 0.05 & 0.865 & -0.07 & 0.07 & 0.270 \\
\hline Cancer $($ reference $=$ no $)$ & & & & & & & & & \\
\hline Yes & 0.14 & 0.20 & 0.494 & 0.05 & 0.07 & 0.484 & 0.12 & 0.07 & 0.106 \\
\hline COPD (reference $=$ no) & & & & & & & & & \\
\hline Yes & -0.03 & 0.08 & 0.749 & 0.16 & 0.06 & 0.009 & -0.07 & 0.07 & 0.257 \\
\hline Ischemic heart disease $($ reference $=$ no) & & & & & & & & & \\
\hline Yes & -0.09 & 0.30 & 0.757 & 0.00 & 0.10 & 0.989 & 0.03 & 0.07 & 0.641 \\
\hline Hypertension (reference $=$ no) & & & & & & & & & \\
\hline Yes & 0.34 & 0.23 & 0.135 & 0.04 & 0.06 & 0.466 & -0.02 & 0.05 & 0.640 \\
\hline Diabetes (reference $=$ no) & & & & & & & & & \\
\hline Yes & -0.31 & 0.23 & 0.181 & 0.05 & 0.06 & 0.352 & -0.05 & 0.05 & 0.345 \\
\hline ICU admission (reference $=$ no) & & & & & & & & & \\
\hline Yes & 1.06 & 0.07 & $<0.001$ & 0.88 & 0.05 & $<0.001$ & 0.85 & 0.06 & $<0.001$ \\
\hline Chest CT (reference $=$ no) & & & & & & & & & \\
\hline Yes & 0.68 & 0.04 & $<0.001$ & 0.48 & 0.03 & $<0.001$ & 0.29 & 0.04 & $<0.001$ \\
\hline ACEI use (reference $=$ no) & & & & & & & & & \\
\hline Yes & - & - & - & -0.26 & 0.32 & 0.421 & -0.15 & 0.18 & 0.422 \\
\hline ARB use (reference $=$ no) & & & & & & & & & \\
\hline Yes & -0.13 & 0.28 & 0.639 & -0.02 & 0.06 & 0.694 & 0.05 & 0.06 & 0.320 \\
\hline Lopinavir/ritonavir use $($ reference $=$ no $)$ & & & & & & & & & \\
\hline Yes & 0.69 & 0.05 & $<0.001$ & 0.64 & 0.03 & $<0.001$ & 0.46 & 0.05 & $<0.001$ \\
\hline HCQ use (reference $=$ no) & & & & & & & & & \\
\hline Yes & 0.40 & 0.05 & $<0.001$ & 0.36 & 0.04 & $<0.001$ & 0.33 & 0.05 & $<0.001$ \\
\hline
\end{tabular}

Note: Multivariate gamma regression model including mechanical ventilator application and interferon use.

Abbreviations: SE, standard error; COPD, chronic obstructive pulmonary disease; ICU, intensive care unit; CT, computed tomography; ACEI, angiotensin-convertingenzyme inhibitor; ARB, angiotensin II receptor blocker; HCQ, hydroxychloroquine.

days) and other respiratory diseases (4 days). ${ }^{23-25,27}$ In Korea and other Asia-Pacific countries, direct medical costs for respiratory diseases were observed to be higher in pharmaceutical and specialist visit sectors than hospitalization, as inpatient treatment occurred infrequently. ${ }^{27}$ However, as the unit cost of hospitalization is generally much higher than that of pharmaceuticals and outpatient visits, diseases that require more inpatient treatment and hospitalization in terms of isolation to prevent further spread of pathogens, such as COVID-19, MERS, and pneumonia, may result in higher medical costs than others. $^{28}$ Moreover, it is notable that COVID-19 patients spent fewer days in hospital and incurred lower medical expenses than pneumonia and MERS patients, which can 
be explained by the lower fatality of COVID-19 cases $(2.4 \%$; May 12,2020$)$ than that of the others in Korea (pneumonia, 3.2\%; MERS, 20.4\%). ${ }^{6,23,24}$ However, indirect costs for social infection prevention and control were not estimated in this study and must be considered through further investigations.

Globally, the mean direct medical expenses in China due to Avian influenza (H7N9) (USD 10,969), seasonal influenza (USD 1797), and SARS (USD 1886) were higher than the estimated cost of COVID-19 patients included in our study (USD 1193.7). ${ }^{17,18}$ In addition, the average cost of medical expenses due to MERS in Saudi Arabia (USD 12,947.03) was also higher than that due to COVID-19. ${ }^{28}$ Lower medical expenses due to COVID-19 compared to the aforementioned diseases may be due to the difference between case fatalities. ${ }^{17}$ In addition, difference in healthcare systems between countries should be considered. To assess the estimated results of medical expenses among Korean patients, further investigations in other countries with different settings are required. To the best of our knowledge, there have been few or no studies globally on the nationwide direct medical expenses due to COVID-19. In a scenario study in the US, the direct medical cost of COVID-19 was predicted to be USD $3045 .^{29}$ In addition, average medical expenses of COVID-19 patients from two hospitals located in China were estimated to be USD 274 for one and USD 1074 for the other. ${ }^{30}$ More research is needed to estimate the healthcare costs of COVID-19 patients, especially in nationally representative samples.

Another branch of findings from this study is the influencing factors of medical expenses and the length of stay in hospital among COVID-19 patients. In Korea, patients with COVID-19 are discharged when they meet either the clinical or the testing criteria. ${ }^{31}$ Symptomatic patients are discharged at improved clinical symptoms without any fever for the minimum of 72 hours after 10 days of symptom onset (clinical criteria) or the same observed 7 days after the onset and getting the negative results in two 24-hour interval PCR-tests in a row. Among the asymptomatic patients, the criteria are the same except that the requirement is not the lost fever and improving symptoms but the absolute symptomless state. Since the patients were discharged not only based on the PCR-tests but majorly considering the improvement and the presence of symptoms, the main variables of interest in the current study, medical expenses and the length of hospital stay, can be proxy indicators for disease severity. In particular, results from our adjusted models showed that female patients, patients aged 0-19 years, healthcare service from providers in Daegu or Gyeongbuk region, lopinavir/ritonavir use, and hydroxychloroquine use were associated with higher medical expenses and longer hospital stays among COVID-19 patients. However, based on observations by age group, providers from Daegu or Gyeongbuk were associated with higher cost of medical expenditures for ages 20-39 years and longer hospital stays among those aged 20-64 years. Comorbid conditions of cancer and ischemic heart disease were strongly associated with shorter hospital stays among COVID-19 patients but became insignificant when analyzed by each age group.

Our findings suggest that among COVID-19 patients, the age group 65 years or above is significantly associated with lower medical expenses and a shorter length of stay in hospital than the age group 0-19 years, supposedly because of the higher fatality and faster death of the older population: In this study, older patients were more likely to report higher medical expenses and longer stay in hospital when considering age as a single factor; however, opposite results were shown in adjusted models with numerous variables. According to a retrospective cohort study in Wuhan, China, the median length of stay in hospital was 11 days per patient in total, 7.5 days among deceased, and 12.0 days among survivors. ${ }^{15}$ Other research from New York reported a shorter length of stay in hospital for non-survivors than survivors among the age group 65 years or above. ${ }^{16}$ Thus, the association between older ages ( $\geq 65$ years) and shorter length of stay in hospital may be due to the higher likelihood of death of older populations and consequent lower medical expenses.

In previous studies, comorbid conditions of COVID-19 were more prevalent among ICU admissions than non-ICU admissions. ${ }^{12}$ In particular, coronary heart disease, diabetes, and hypertension were risk factors for in-hospital deaths among COVID-19 patients. ${ }^{15}$ In addition, length of stay in hospital was longer for deceased diabetic patients than for deceased non-diabetic ones, and for deceased patients with hypertension than for deceased nonhypertensive patients. ${ }^{16}$ The results from our study showed that patients presenting comorbid conditions of cancer and ischemic heart disease had higher odds of shorter stay in hospital when adjusted for age, but when analyzed separately by age group or in crude analyses for each comorbid condition as a single variable, there was no significant 
association between the prevalence of comorbidities and outcome indicators. All other comorbid diseases had no significant association with medical expenses or length of stay in hospital for COVID-19 patients. Previously, high prevalence of comorbidities in the elderly was considered to be able to explain the vulnerability of the older population to COVID-19. ${ }^{32,33}$ Our results from analyses in different age groups suggest the opposite: the association between comorbid conditions and undesired outcomes (eg, deaths or ICU admissions) could be due to the older age of comorbidity-prevalent groups.

Another comorbid condition considered in our study was the use of RAAS inhibitors, including ACEI and ARB. We find that ACEI and ARB usage among COVID-19 patients is not significantly related to an increase in medical expenses or length of stay in hospital. Similarly, several studies have reported insignificant associations between ACEI/ARB and undesired clinical outcomes. ${ }^{14,16}$ Although the biphasic effects of ACEI/ ARB are still being investigated, our study supports the view that there is no need to eliminate or change the drugs for COVID-19 patients. $^{34}$

In our study, anti-viral treatments with lopinavir/ritonavir and hydroxychloroquine were significantly related to high medical expenses and longer hospital stays among COVID-19 patients. Association between anti-viral drugs and high medical costs or longer hospitalization could be explained by usual treatment procedures whereby antivirals are used to treat severe patients; for example, the median time from the onset of illness to lopinavir/ritonavir prescription was 14 days in a previous study. ${ }^{15}$ Therefore, the results from this study do not necessarily imply that the drugs should not be used; however, neither do they support the efficacy of lopinavir/ritonavir and hydroxychloroquine usage.

Our study has some limitations. First, due to the nature of insurance benefit claims data, only the information that could be reported in the claims statements could be included, resulting in missing "death" variable. As a compensation, we considered medical expenses and the length of stay in hospital as our outcome variables rather than the fixed clinical outcomes of discharged alive or dead. Second, owing to the nature of insurance benefit claims data, the timeline of each provider's claims might not precisely coincide with the point of event occurrence in each patient, as the data depend solely on providers' claims to the NHI. Therefore, there is a limitation of missing data from non-claimed healthcare utilization that is not-yet-to-be-claimed. However, the actual analyzed period included in the study can be inferred from the number of confirmed patients and methods of provider claims. Health insurance claims should be claimed weekly; however, in cases of patients with prolonged hospitalization, providers disperse claims benefit in monthly installments. Corroborating, the number of participants included in the study was 7590 (claimed as of May 15, 2020), which is close to the number as of March 11, 2020 (7755). ${ }^{6}$ Therefore, it can be reasonably said that the actual period included in the study was until early March, reducing the uncertainty due to the missing data. Finally, as the pandemic is ongoing, our study results are provisional; the average costs and the length of in-hospital stay are likely to be changed. However, there is a clear need for the estimation of current status despite the changes likely being made in the future, as it could support the decision making process while enabling the horizontal (ie, between countries) and vertical (ie, between early and later phases of the pandemic) comparisons.

\section{Conclusion}

Despite the limitations, to the best of our knowledge, this is the first study to estimate the cost of direct medical expenses and the length of stay in hospital among COVID-19 patients using a nationwide database. In particular, as far as we know, no previously published research has calculated the direct cost of healthcare utilization due to COVID-19 nationally using administrative data that consists the entire population. Our findings could serve as a global reference for direct medical expenses and length of hospital stay among COVID-19 patients. Furthermore, this study provides evidence of disease severity mostly influenced by age of COVID-19 patients above any other factors; proxy indicators of the disease severity (ie, medical expenditures and duration of hospital stay) were not significantly associated with variables previously perceived or debated to worsen clinical outcomes including comorbidities, antiviral use, and use of ACEI and ARB. Given that the outcome of COVID-19 patients is not greatly influenced by significant presenting characteristics, response to the disease should focus on infection prevention rather than targeted clinical measures after infection.

\section{Acknowledgments}

The authors appreciate the healthcare professionals treating COVID-19 patients in Korea, and the Ministry of 
Health and Welfare and the Health Insurance Review \& Assessment Service of Korea for sharing invaluable national health insurance claims data in a prompt manner.

\section{Funding}

This study was supported by a grant from the Korean Health Technology R\&D Project through the Korean Health Industry Development Institute (KHIDI), funded by the Ministry of Health and Welfare, Republic of Korea (grant no. HI18C0446).

\section{Disclosure}

All of the authors report no conflicts of interest in this work.

\section{References}

1. Kim JY, Choe PG, Oh Y, et al. The first case of 2019 novel coronavirus pneumonia imported into Korea from Wuhan, China: implication for infection prevention and control measures. J Korean Med Sci. 2020;35(5):e61. doi:10.3346/jkms.2020.35.e61

2. Korea Centers for Disease Control \& Prevention. Updates on COVID-19 in Republic of Korea. September 14, 2020. Available from: https://is.cdc.go.kr/upload_comm/syview/doc.html?fn= 160007467707700.pdf\&rs=/upload_comm/docu/0030/. Accessed October 10, 2020.

3. Korea Centers for Disease Control \& Prevention. Updates on COVID-19 in Republic of Korea. March 20, 2020. Available from: https://is.cdc.go.kr/upload_comm/syview/doc.html?fn= 158468802182900.pdf\&rs=/upload_comm/docu/0030/. Accessed October 10, 2020.

4. Korean Society of Infectious Diseases and Korea Centers for Disease Control and Prevention. Analysis on 54 Mortality Cases of Coronavirus Disease 2019 in the Republic of Korea from January 19 to March 10, 2020. J Korean Med Sci. 2020;35(12): e132. doi:10.3346/jkms.2020.35.e132

5. Kim DH, Choe YJ, Jeong JY. Understanding and interpretation of case fatality rate of coronavirus disease 2019. J Korean Med Sci. 2020;35(12):e137. doi:10.3346/jkms.2020.35.e137

6. Statistics Korea. Population Projections for Korea. Available from: https://kosis.kr/eng/statisticsList/statisticsListIndex.do?menuId=M_ 01_01\&vwcd=MT_ETITLE\&parmTabId=M_01_01\&statId= $1962005 \&$ themaId $=\#$ SelectStatsBoxDiv. Accessed February 23, 2021.

7. Statistics Korea. Statistics of Residence Registration Population. Available from: https://kosis.kr/statisticsList/statisticsListIndex.do? parentId=A.1\&vwcd=MT_ZTITLE\&menuId=M_01_01\#contentgroup. Accessed February 23, 2021.

8. Rhee EJ. Prevalence and current management of cardiovascular risk factors in Korean adults based on fact sheets. Endocrinol Metab. 2020;35(1):85-94. doi:10.3803/EnM.2020.35.1.85

9. Park H, Jung SY, Lee K, et al. Prevalence of chronic obstructive lung disease in Korea using data from the fifth Korea national health and nutrition examination survey. Korean J Fam Med. 2015;36 (3):128-134. doi:10.4082/kjfm.2015.36.3.128

10. Peng F, Tu L, Yang Y, et al. Management and treatment of COVID-19: the Chinese experience. Can J Cardiol. 2020;36 (6):915-930. doi:10.1016/j.cjca.2020.04.010

11. Huang C, Wang Y, Li X, et al. Clinical features of patients infected with 2019 novel coronavirus in Wuhan, China. Lancet. 2020;395 (10223):497-506. doi:10.1016/S0140-6736(20)30183-5
12. Wang D, Hu B, Hu C, et al. Clinical characteristics of 138 hospitalized patients with 2019 novel coronavirus-infected pneumonia in Wuhan, China. JAMA. 2020;323(11):1061-1069. doi:10.1001/jama.2020.1585

13. Wan S, Xiang Y, Fang W, et al. Clinical features and treatment of COVID-19 patients in northeast Chongqing. J Med Virol. 2020;92 (7):797-806. doi:10.1002/jmv.25783

14. Guo T, Fan Y, Chen M, et al. Cardiovascular implications of fatal outcomes of patients with coronavirus disease 2019 (COVID-19). JAMA Cardiol. 2020;5(7):811. doi:10.1001/jamacardio.2020.1017

15. Zhou F, Yu T, Du R, et al. Clinical course and risk factors for mortality of adult inpatients with COVID-19 in Wuhan, China: a retrospective cohort study. Lancet. 2020;395(10229):1054-1062. doi:10.1016/S0140-6736(20)30566-3

16. Richardson S, Hirsch JS, Narasimhan M, et al. Presenting characteristics, comorbidities, and outcomes among 5700 patients hospitalized with COVID-19 in the New York City area. JAMA. 2020;323 (20):2052. doi:10.1001/jama.2020.6775

17. Huo X, Chen -L-L, Hong L, et al. Economic burden and its associated factors of hospitalized patients infected with A (H7N9) virus: a retrospective study in Eastern China, 2013-2014. Infect Dis Poverty. 2016;5(1):79. doi:10.1186/s40249-016-0170-5

18. Zhou L, Situ S, Huang T, et al. Direct medical cost of influenza-related hospitalizations among severe acute respiratory infections cases in three provinces in China. PLoS One. 2013;8(5): e63788. doi:10.1371/journal.pone.0063788

19. Feng D, Jia N, Fang L-Q, et al. Duration of symptom onset to hospital admission and admission to discharge or death in SARS in mainland China: a descriptive study. Trop Med Int Health. 2009;14:28-35. doi:10.1111/j.1365-3156.2008.02188.x

20. National Health Insurance Service. National Health Insurance System of Korea. 2015. Availablefrom: https:/www.nhis.or.kr/static/html/ wbd/g/a/wbdga0704.html. Accessed May 19, 2020.

21. Ministry of Health and Welfare, Health Insurance Review \& Assessment Service. Guidelines for COVID-19International Research Co-hosted by MoHW and HIRA of Korea (ver.1). 2020. Available from: https://hira-covid19.net/view_community?commu nity_id=1585292525339. Accessed May 19, 2020.

22. Bank of Korea Economic Statistics System. 8.8.1.1 Arbitraged Rates of Major Currencies Against Won. May 15, 2020. Available from: http://ecos.bok.or.kr/. Accessed May 19, 2020.

23. Yoo KH, Yoo CG, Kim SK, et al. Economic burden and epidemiology of pneumonia in Korean adults aged over 50 years. $J$ Korean Med Sci. 2013;28(6):888-895. doi:10.3346/jkms.2013.28.6.888

24. Ministry of Health and Welfare. The 2015 MERS Outbreak in the Republic of Korea: Learning from MERS. Sejong: Ministry of Health and Welfare; 2016.

25. Kim C, Kim Y, Yang DW, et al. Direct and indirect costs of chronic obstructive pulmonary disease in Korea. Tuberc Respir Dis. 2019;82 (1):27-34. doi: $10.4046 / \operatorname{trd} .2018 .0035$

26. Lee Y-H, Yoon S-J, Kim E-J, et al. Economic burden of asthma in Korea. Allergy Asthma Proc. 2011;32(6):35-40. doi:10.2500/aap.20 11.32.3479

27. Wang Y, Ghoshal AG, Bin AA, et al. Quality of life and economic burden of respiratory disease in Asia-Pacific-Asia-Pacific burden of respiratory diseases study. Value Health Reg Issues. 2016;9:72-77. doi:10.1016/j.vhri.2015.11.004

28. AlRuthia Y, Somily AM, Alkhamali AS, et al. Estimation of direct medical costs of Middle East respiratory syndrome coronavirus infection: a single-center retrospective chart review study. Infect Drug Resist. 2019;12:3463-3473. doi:10.2147/IDR.S231087

29. Bartsch SM, Ferguson MC, McKinnel JA, et al. The potential health care costs and resource use associated with COVID-19 in the United States. Health Aff. 2020;39(6):1-7. doi:10.1377/hlthaff.2020.00426

30. Yan F, Zhang X, Zhang M, et al. Direct medical expense of COVID-19 patients at Fangcang shelter hospital and Leishenshan designated hospital in Wuhan, China. SSRN [Preprint]. 2020. 
31. Coronavirus Disease-19, Republic of Korea. Patient Treatment \& Management. Discharge. June 25, 2020. Available from: http://ncov. mohw.go.kr/en/baroView.do?brdId=11\&brdGubun= $112 \&$ dataGubun $=\&$ ncvContSeq $=\&$ contSeq $=\&$ board_id $=\&$ gubun $=$. Accessed February 28, 2021.

32. Yang J, Zheng Y, Gou X, et al. Prevalence of comorbidities in the novel Wuhan coronavirus (COVID-19) infection: a systematic review and meta-analysis. Int $J$ Infect Dis. 2020;94:91-95. doi:10.1016/j. ijid.2020.03.017
33. Zhang J-J, Dong X, Cao Y-Y, et al. Clinical characteristics of 140 patients infected with SARS-CoV-2 in Wuhan, China. Allergy. 2020:1-12. doi:10.15036/arerugi.69.1

34. Jung SY, Choi JC, You SH, et al. Association of renin-angiotensinaldosterone system inhibitors with COVID-19-related outcomes in Korea: a nationwide population-based cohort study. Clin Infect Dis. 2020. doi:10.1093/cid/ciaa624

\section{Publish your work in this journal}

Risk Management and Healthcare Policy is an international, peerreviewed, open access journal focusing on all aspects of public health, policy, and preventative measures to promote good health and improve morbidity and mortality in the population. The journal welcomes submitted papers covering original research, basic science, clinical \& epidemiological studies, reviews and evaluations, guidelines, expert opinion and commentary, case reports and extended reports. The manuscript management system is completely online and includes a very quick and fair peer-review system, which is all easy to use. Visit http://www.dovepress.com/testimonials.php to read real quotes from published authors.

Submit your manuscript here: https://www.dovepress.com/risk-management-and-healthcare-policy-journal 